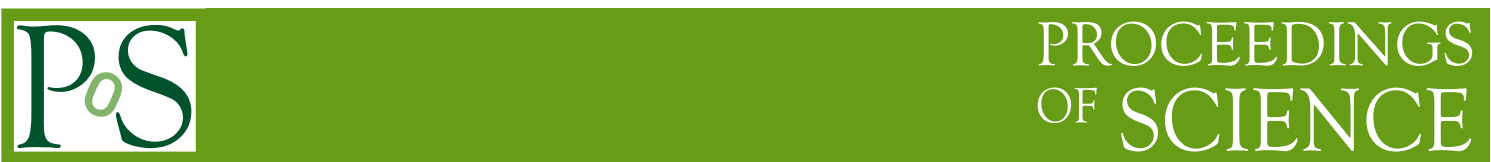

\title{
SUSY global fits and recast of ATLAS searches at 13 TeV.
}

\author{
Isabel Suárez Fernández* (on behalf of the MasterCode Collaboration) \\ University of Santiago de Compostela (USC) \\ Galician Institute of High Energy Physics (IGFAE) \\ E-mail: isfer@mundo-r.com
}

In this work, we essentially show how we recast analysis and data from the ATLAS experiment searches at $13 \mathrm{TeV}$ with the objective of using them, subsequently, in other supersymmetric models. As the recasting of ATLAS data will be a very relevant input to carry out future global fits of supersymmetric models, as an example, we show the application of this recast to CMSSM.

The European Physical Society Conference on High Energy Physics

5-12 July, 2017

Venice

${ }^{*}$ Speaker. 


\section{Direct searches of supersymmetry in ATLAS at $\sqrt{s}=13 \mathrm{TeV}$ [1]}

LHC recast is a project we are developing for the MasterCode collaboration. Its aim is to build a code to recast ATLAS and CMS searches in other SUSY models. In this case, work in the same simplified model as ATLAS in [2] and we show how to reproduce their mass constraint and exclusion plot to test our algorithm.

\subsection{Final states with two same sign leptons or three leptons using $13.2 \mathbf{f b}^{-1}$ at $\sqrt{s}=13 \mathrm{TeV}$}

We work in the topology corresponding to final states with more than three leptons [2], where $m\left(\tilde{\chi}_{2}^{0}\right)$ and $m(\tilde{l} / \tilde{v})$ are fixed relative to $m(\tilde{g})$ and $m\left(\tilde{\chi}_{1}^{0}\right)$. We have carried out analysis for 9 different topologies using 32 different signal regions, however, in this proceedings we focus on the gluino topology shown in figure (1) as an illustration.

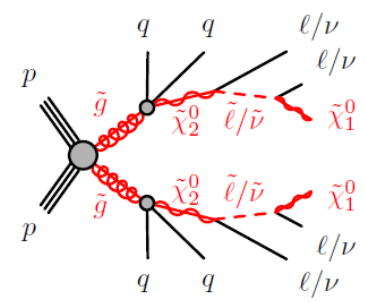

Figure 1: SUSY topology with a final state of three or more leptons.

\subsection{Simulating the efficiency}

Fixing $m\left(\tilde{\chi}_{2}^{0}\right)$ and $m(\tilde{l} / \tilde{v})$ relative to $m(\tilde{g})$ and $m\left(\tilde{\chi}_{1}^{0}\right)$, the efficiency is a function of the two last masses. We first estimate the efficiency using the following procedure. We use an experimental simulation of pp collisions based on Pythia 8.2 [3]. After generating the events, we use Delphes 3 [4], a software that performs a multipurpose detector and that we use to recreate the ATLAS detector. On top of the Delphes simulation, we apply the selection requirements of each signal region. Finally, we simulate the efficiency for a wide range of the masses in order to be able to compute a likelihood for each pair of $m(\tilde{g})$ and $m\left(\tilde{\chi}_{1}^{0}\right)$.

\subsection{Likelihood}

The profiled Likelihood is given by

$$
\mathscr{L}\left(N_{o b s} \mid S_{\text {exp }}\right)=\frac{1}{N_{o b s} !} e^{-\left(S_{\text {exp }}+\hat{b}\right)} e^{-\frac{1}{2}\left(\frac{\hat{b}-b_{0}}{\sigma(b)}\right)^{2}}\left(\hat{b}+S_{\text {exp }}\right)^{N_{o b s}}
$$

with

$$
\hat{b}=\frac{\left(b_{0}-S_{\text {exp }}-\sigma^{2}(b)\right)}{2}+\frac{1}{2}\left[\left(b_{0}-S_{\exp }-\sigma^{2}(b)\right)^{2}+4\left(\sigma^{2}(b) N_{\text {obs }}-S_{\exp } \sigma^{2}(b)+S_{\text {exp }} b_{0}\right)\right]^{\frac{1}{2}} .
$$

In these expressions $N_{o b s}$ is the number of observed events and $b_{0}$ is the SM background, both of which are reported in [2]. The expected signal is given as $S_{\exp }=\sigma \varepsilon L$, where $\sigma$ is the production 
cross-section, $\varepsilon$ is the efficiency and $L$ is the luminosity. Since the cross-section and the efficiency are functions of SUSY masses, the Likelihood has an indirect dependence on $m(\tilde{g})$ and $m\left(\tilde{\chi}_{1}^{0}\right)$ trough $S_{\text {exp }}$.

\subsection{Constraint and exclusion plot}

We compute the probability for a wide range of input masses following the steps defined above. With these results we represent the 2D exclusion plot of figure (2.b). In the axes we have our inputs. The gluino mass $(m(\tilde{g}))$ on the $\mathrm{X}$ axis and the lightest neutralino mass $\left(m\left(\tilde{\chi}_{1}^{0}\right)\right)$ in the $\mathrm{Y}$ axis, both expressed in $\mathrm{GeV}$. The color lines represent the probability of the process happening, where the darkest blue represents the exclusion at $95 \%$. We compare our exclusion limit with the one obtained by ATLAS, which is the red solid line shown in figure (2.a). One can see these limits are in a good argeement.

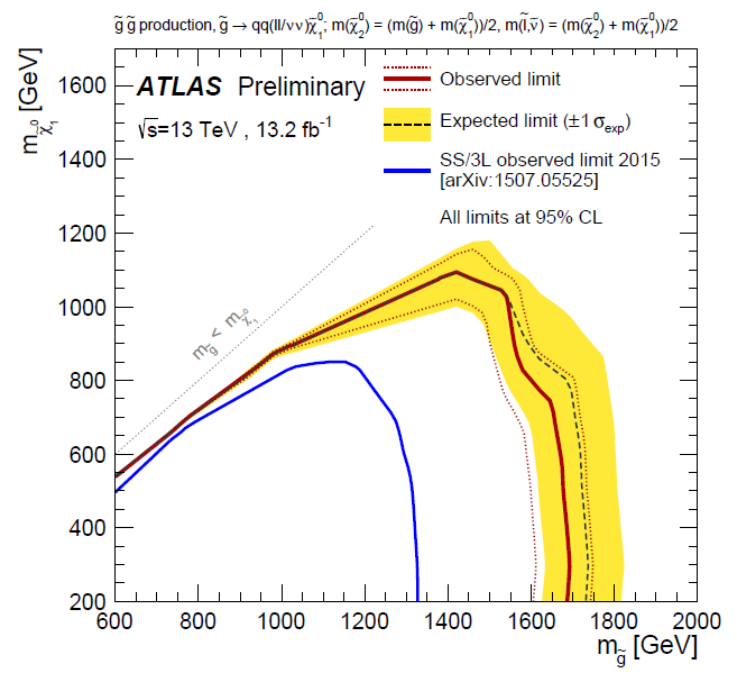

(a)

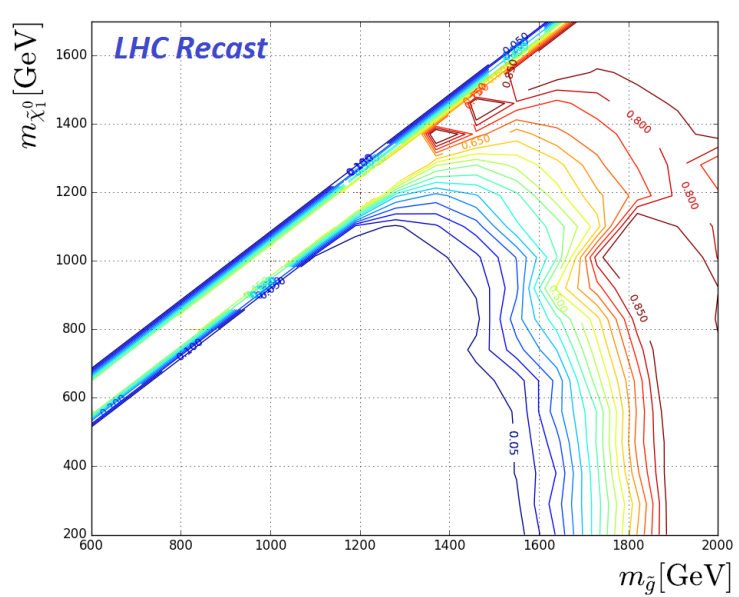

(b)

Figure 2: 2D $\chi^{2}$-probability distribution of the first analysis of [2]. On the left side the exclusion contour obtained by ATLAS is shown. On the right side the one obtained using our procedure is shown, where the color lines indicate the $\chi^{2}$ probability, the low mass region being excluded at 95\% CL.

\section{Application to CMSSM}

The efficiencies and mass constraints we obtained in the previous analysis were for simplified models, rather than full SUSY models. The next step is recasting these searches in different SUSY models, such as the Constrained Minimal Supersymmetric Standard Model (CMSSM) we show in this case. In this model important parameters are the common mass of the scalars $\left(m_{0}\right)$ and the common mass of the gauginos $\left(m_{1 / 2}\right)$, defined at the unification scale $\left(\sim 10^{16} \mathrm{GeV}\right)$. We derive a constraint in the $\left(m_{0}, m_{1 / 2}\right)$ plane using the procedure explained above. We assume the same value of the other parameters of $\operatorname{CMSSM}\left(A_{0}=-3440, \beta=21\right)$ as at the previous MasterCode best fit point [5]. 
In figure (3) we can see the exclusion plot and the $\chi^{2}$ probability distribution for the $\left(m_{0}, m_{1 / 2}\right)$ plane in the CMSSM. The green star is the previous best fit point from [5] and it is clear that the new one should be displaced to a region with higher probability. Again the darkest blue means exclusion at 95\% CL, therefore, we see that the low energy region is excluded in this model. In fact, this exclusion is stronger than in previous results.

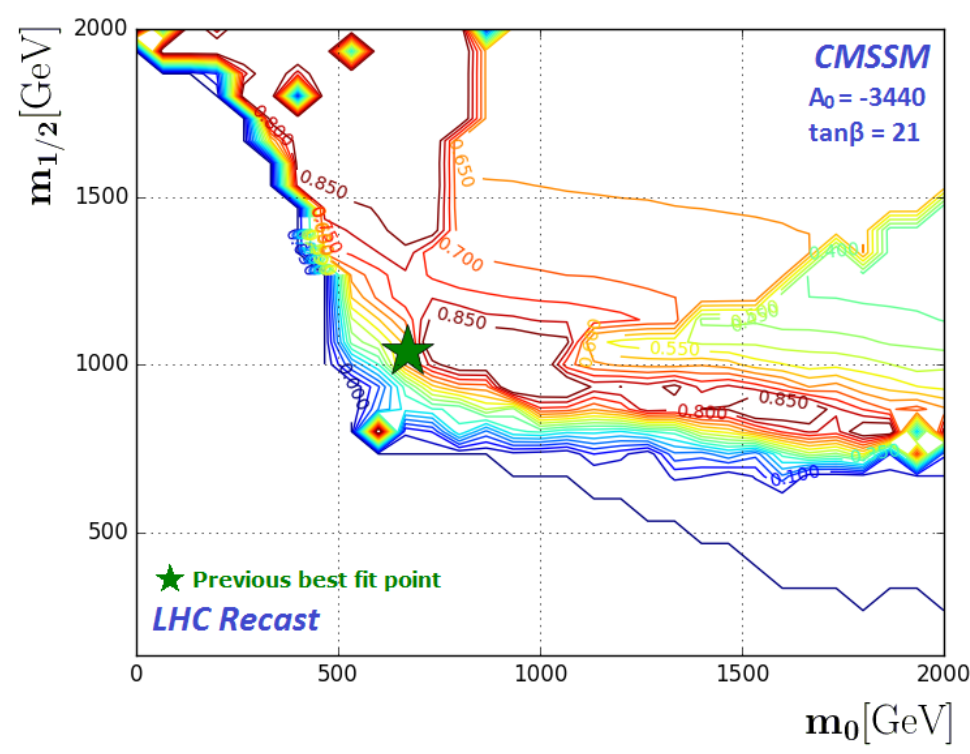

Figure 3: Exclusion contour and $\chi^{2}$-probability distribution for $m_{0}$ and $m_{1 / 2}$ in the CMSSM.

\section{Conclusions}

We focused on the direct SUSY searches of ATLAS at $\sqrt{s}=13 \mathrm{TeV}$ with the latest experimental data. We developed a software to analyze in the simplified model that ATLAS proposes the SUSY processes that lead us to the mass constraint and exclusion contour we have shown above as well as others not displayed in this summary. With this, we were able to reproduce ATLAS results and developed a tool to use later in other more real SUSY models as CMSSM. This recast will be also used in more SUSY models as pMSSM, NUHM and SU(5) GUT's.

\section{References}

[1] ATLAS collaboration. Atlas supersymmetry (susy) searches. https://twiki.cern.ch/ twiki/bin/view/AtlasPublic/SupersymmetryPublicResults\#13_TeV_data, 2017.

[2] The ATLAS collaboration. Search for supersymmetry with two same-sign leptons or three leptons using $13.2 \mathrm{fb}^{-1}$ of $\sqrt{s}=13 \mathrm{TeV} p p$ collision data collected by the ATLAS detector. 2016.

[3] Torbjörn Sjöstrand, Stefan Ask, Jesper R. Christiansen, Richard Corke, Nishita Desai, Philip Ilten, Stephen Mrenna, Stefan Prestel, Christine O. Rasmussen, and Peter Z. Skands. An Introduction to PYTHIA 8.2. Comput. Phys. Commun., 191:159-177, 2015. 
[4] J. de Favereau, C. Delaere, P. Demin, A. Giammanco, V. Lemaître, A. Mertens, and M. Selvaggi. Delphes 3: a modular framework for fast simulation of a generic collider experiment. Journal of High Energy Physics, 2014(2):57, Feb 2014.

[5] O. Buchmueller et al. The CMSSM and NUHM1 after LHC Run 1. Eur. Phys. J., C74(6):2922, 2014.

[6] Search for squarks and gluinos in events with an isolated lepton, jets and missing transverse momentum at $\sqrt{s}=13 \mathrm{TeV}$ with the ATLAS detector. Technical Report ATLAS-CONF-2016-054, CERN, Geneva, Aug 2016.

[7] Georges Aad et al. Search for gluinos in events with an isolated lepton, jets and missing transverse momentum at $\sqrt{s}=13$ Te V with the ATLAS detector. Eur. Phys. J., C76(10):565, 2016.

[8] Georges Aad et al. Search for supersymmetry at $\sqrt{s}=13 \mathrm{TeV}$ in final states with jets and two same-sign leptons or three leptons with the ATLAS detector. Eur. Phys. J., C76(5):259, 2016.

[9] Morad Aaboud et al. Search for squarks and gluinos in final states with jets and missing transverse momentum at $\sqrt{s}=13 \mathrm{TeV}$ with the ATLAS detector. Eur. Phys. J., C76(7):392, 2016.

[10] Chunhui Chen. New approach to identifying boosted hadronically-decaying particle using jet substructure in its center-of-mass frame. Phys. Rev., D85:034007, 2012.

[11] The ATLAS collaboration. Search for electroweak production of supersymmetric tau leptons in $\sqrt{s}=13 \mathrm{TeV} p p$ collisions with the ATLAS detector. 2016.

[12] The ATLAS collaboration. Search for supersymmetry with two and three leptons missing transverse momentum in the final state at $\sqrt{s}=13 \mathrm{TeV}$ with the ATLAS detector. 2016.

[13] Diego Martínez Santos. Study of the very rare decay $B_{s} \rightarrow \mu^{+} \mu^{-}$in $L H C b$. PhD thesis, Universidade de Santiago de Compostela.

[14] Kees Jan de Vries. Global Fits of Supersymmetric Models after LHC Run 1. PhD thesis, Imperial College London.

[15] Alan Barr, Christopher Lester, and P. Stephens. m(T2): The Truth behind the glamour. J. Phys., G29:2343-2363, 2003.

[16] The mastercode project. http://mastercode.web. cern. ch/mastercode/, 2017. 